\title{
La invisibilización de las mujeres y la construcción de identidades masculinas en los discursos políticos de las campañas electorales de 1953 y 1958 en Costa Rica
}

\author{
Manuel Gamboa Brenes ${ }^{1}$
}

Recepción: 31 de octubre de 2013 / Aprobación: 11 de diciembre de 2013

\section{Resumen}

Este artículo examina la invisibilización de las mujeres en los discursos políticos en las campañas electorales de 1953 y 1958 en Costa Rica para determinar la construcción de identidades de género después de la Guerra Civil de 1948 y del derecho al voto a las mujeres. Mediante el análisis comparativo de la propaganda política de cada partido en los periódicos del país antes de cada elección presidencial, se identifican las principales características de los discursos dirigidos hacia las mujeres buscando las posibles intenciones de quienes construyeron esos discursos. Además, se estudian los discursos que privilegian el papel de los hombres durante la Guerra Civil de 1948, para buscar la construcción de identidades de masculinidad que determinarían el tipo de política hecha en la década de 1950 en Costa Rica. El estudio concluye que los intereses de los actores políticos provocaron que la Guerra Civil de 1948 recuperada en los discursos políticos durante la década de 1950 fuera un asunto de hombres donde las mujeres ocupaban un papel secundario.

\section{Palabras clave}

Género, identidades, discursos políticos, campaña electoral, Guerra Civil de 1948

\section{Abstract}

This article examines the invisibilization of women in political discourse in Costa Rica's campaigns of 1953 and 1958 in order to determine the way in which gender identities is built after the Civil War of 1948 and women's right to vote. Through the comparative analysis of the political propaganda of each party which appeared in major newspapers in the country before each presidential election, identified the main characteristics of the speech directed to or towards women looking for them the possible intentions of those who were building these discourses. It also explores the main features of the speeches that privilege men's role during the Civil War of 1948, to find

1 Costarricense. Licenciado en Historia por la Universidad de Costa Rica, Costa Rica. Investigador del Museo Histórico y Tecnológico del Instituto Costarricense de Electricidad, Costa Rica. Correo electrónico: manuelgamboacr@hotmail.com 
in them the construction of identities of masculinity which would determine the type of policy making in the 1950s in Costa Rica. The study concludes that the interests of the political actors provoked that the Civil War of 1948 recovered in the political speeches during the decade of 1950 was a men's matter where the women were occupying a secondary paper.

\section{Key words}

Gender, identities, political discourse, electoral campaign, Civil War

\section{Resumo}

Este artigo examina a invisibilidade das mulheres no discurso político nas campanhas de 1953 e 1958 na Costa Rica, para determinar a construção da identidade de gênero após a Guerra Civil de 1948 e o direito ao voto das mulheres. Através da análise comparativa da propaganda política de cada partido nos jornais do país antes de cada eleição presidencial, se identificou as principais características dos discursos dirigidos às mulheres buscando as possíveis intenções daqueles que construíram esses discursos. Além disso, se estuda os discursos que privilegiam o papel dos homens durante a Guerra Civil de 1948 para buscar a construção de identidades de masculinidade que determinariam o tipo de política feita na década de 1950 na Costa Rica. O estudo conclui que os interesses dos atores políticos provocaram que a Guerra Civil de 1948, recuperada nos discursos políticos durante a década de 1950, fosse um assunto de homens, onde as mulheres ocupavam um papel secundário.

\section{Palabras clave}

Gênero, identidades, discursos políticos, campanha eleitoral, Guerra Civil

\section{Introducción}

a década de 1950 puede ser considerada muy importante para la incorporación de la mujer costarricense en la política del país. Efectivamente, a partir de la ley que establece el voto femenino en 1949 (Rodríguez, 2003a, 17-29) las mujeres se convierten en un elemento importante en los procesos político-electorales del país por varias razones lógicas. En primer lugar, las mujeres ampliaban en casi un cien por ciento el padrón electoral, lo que definitivamente todos los candidatos debían tomar en consideración en sus discursos políticos. La elección de los puestos políticos dejaba de ser un asunto exclusivo para hombres, y ello suponía un problema a la vez que una ventaja potencial para los intereses de cada partido político. En segundo lugar, aunque las mujeres tenían una historia de lucha muy importante para adquirir la igualdad política y del voto, con él también se iniciaba un lento y complicado proceso de incorporación real de la mujer a los puestos políticos en el país. 
En efecto, con la conquista del voto femenino se avanzó en el proceso de construcción de condiciones más igualitarias de participación y transparencia electoral. No está de más señalar que las luchas sociales que han estado detrás de cada una de las reformas electorales normalmente han sido invisibilizadas en el mayor de los casos. En este sentido, señala el libro de Macarena Barahona Riera sobre las sufragistas, que la invisibilización de la mujer como sujeto político no ha sido solo un problema de las instituciones políticas de Costa Rica, sino también lo sería durante bastante tiempo en diversos estudios políticos de las ciencias sociales del país. Para Barahona, el estudio de la lucha por el voto femenino es una manera de rescatar ese elemento político y también ideológico de la mujer costarricense (Barahona, 14,15). Para la historiadora Eugenia Rodríguez Sáenz, la evolución de los movimientos feministas y sufragistas en la primera mitad del siglo XX en Costa Rica, deben entenderse en el marco de una serie de factores más amplios como la efervescencia política, las reformas electorales, las políticas sociales implementadas por el Estado liberal, en la redefinición de un sistema ideológico de género, y el impacto que tuvieron las luchas feministas internacionales a nivel local (Rodríguez, 2002, 87).

Por otra parte, en este artículo se argumenta que la década de 1950, además, significó el comienzo de una restructuración socio-política y económica de Costa Rica a raíz de la Guerra Civil de 1948. A primera vista, la forma de hacer política en Costa Rica se modificó a partir de los cambios en la legislación electoral que inician apenas se apaga el fuego de las armas. Empero, lo que en realidad se modificó como resultado del conflicto del 48 fue la aparición de nuevos actores políticos y sociales que capitalizaron sus triunfos de la guerra por medio de políticas electorales y económicas tendientes a consolidar su nueva posición privilegiada (Rovira, 1983). Entonces, consideramos que la manera en que las mujeres son incluidas en el juego político en todas sus formas y posibilidades tendrá mucho que ver con la manera en que estos "nuevos" políticos asumen las reivindicaciones feministas acumuladas hasta ese momento, para hacerlas calzar con sus intereses políticos-electorales.

Otro aspecto, que sí está muy latente en esta década de 1950, es la memoria del conflicto armado y su uso como herramienta electoral por parte de los partidos políticos. Este uso electoral del pasado relacionado a la coyuntura de 1948 se realiza sobre todo para establecer derechos y limitaciones al poder político según sea la lectura de la guerra y claro está, los intereses de una u otra agrupación política (Gamboa, 2012, 56). Por ello, en este artículo planteamos que es necesario establecer una relación analítica entre el tema del voto femenino y la incorporación o no de la mujer en el discurso político relacionado al 48, con el fin de encontrar algunas pistas sobre el trasfondo del cálculo político electoral de los actores políticos que incorporaron a la mujer con su derecho al voto.

Por último, este artículo se inspira en los planteamientos del libro de Alfonso González, quien afirma que es importante tomar en consideración el proceso 
de redefinición de la masculinidad y la feminidad de la sociedad costarricense después de la guerra de 1948, sobre todo el tema relacionado a los ideales de masculinidad que se imponen en los discursos y los comportamientos político-electorales durante la década de 1950 (González, 2005,31-34). Eso sí, los cambios que evidentemente ocurrieron en la sociedad costarricense después del conflicto armado, deben ser entendidos tomando en consideración los elementos que son continuos antes y después del mismo. De ahí que aspectos como amistades y enemistades políticas, personalismo político, patriarcalismo, alianzas y traiciones políticas, son factores que merecen ser analizados sin poner directamente a la guerra del 48 como un punto de referencia. Esto para comprender cómo elementos de la política anterior al 48 afectan y permean a la Costa Rica posterior (Solís, 2006, 191-225).

Este artículo forma parte de una investigación más amplia, realizada sobre la recuperación de la memoria de la Guerra Civil de 1948 en los discursos políticos en las campañas electorales entre 1953 y 1970 (Gamboa, 2012). Haciendo una reinterpretación de las fuentes, el presente ejercicio se basó en el estudio del discurso político-electoral en los artículos publicados en los periódicos La Nación, La República y Diario de Costa Rica las semanas anteriores a las elecciones presidenciales del 26 de julio de 1953 y el 2 de febrero de 1958. De todos los artículos recuperados para el trabajo anterior, se seleccionaron los artículos que contenían un discurso dirigido hacia, o acerca de las mujeres, sobre todo aquellos donde se rescata la memoria de la Guerra Civil de 1948. También se hizo un análisis de aquellos que están relacionados directamente al protagonismo de los hombres durante el mismo conflicto armado. Así, el objetivo es realizar una interpretación discursiva de los contenidos políticos de los textos, además, generar un debate sobre el papel de la masculinidad y feminidad en los cambios políticos sociales originados por la Guerra Civil de 1948.

Este trabajo se propone analizar, por qué las mujeres fueron invisibilizadas en la recuperación de la memoria de la Guerra Civil de 1948, en los discursos de las campañas electorales de 1953 y 1958 en Costa Rica. Además, analiza las posibles razones que originan que en estos discursos sean resaltados el carácter masculino y los ideales de masculinidad durante las campañas electorales. Más específicamente, a partir del estudio del discurso políticoelectoral, se analiza el contenido de los discursos políticos relacionados a la coyuntura de 1948, en el que aparece un discurso dirigido hacia, o sobre las mujeres, para identificar cuál es la imagen de la mujer que los partidos políticos reconstruyen. Por otro lado, se busca identificar también el carácter masculino de los discursos políticos sobre el 48, para estudiar, de qué manera los ideales de masculinidad se imponen en la recuperación de la memoria sobre la Guerra Civil de 1948.

El artículo está dividido en dos secciones. La primera se encarga de mostrar las principales características y particularidades de los discursos políticos sobre mujeres o dirigidos a ellas y con alguna relación a la Guerra Civil de 
1948. Esta sección señala que el discurso elaborado sobre las mujeres y el que es construido por mujeres tendió a rescatar el carácter de contenedoras de la violencia de los hombres, considerando que lo más mencionado en esos discursos es el peligro que las mujeres pierdan a "sus hombres" en un nuevo conflicto armado.

La segunda sección trata de explicar las intenciones de los hombres de la política en sus discursos durante las campañas electorales de 1953 y 1958. Se considera que las necesidades de estos hombres por construir su identidad masculina y política en un contexto de continuidades que trascienden la Guerra Civil de 1948, originan que la lucha electoral no deje de ser un asunto meramente de hombres.

A nivel general, el trabajo logra mostrar que las mismas particularidades de los discursos políticos que invisibilizan a las mujeres como actores políticos con derechos adquiridos con el voto femenino, demuestran que, al momento de hacer la reforma electoral, se estaba muy lejos de tener la intención de incorporar definitivamente a las mujeres en el juego de la política. Lo que se presenta en realidad es un juego de hombres construyendo su propio pasado y devenir político, quienes, con ese fin, utilizan un discurso sobre y para las mujeres que les ayude a vencer a sus contrincantes de turno.

\section{Las mujeres en los discursos políticos sobre el 48: madres, esposas, hermanas e hijas de los combatientes.}

El 28 de agosto del 2013, el Ministerio de la Condición de la Mujer celebró el 60 aniversario del derecho al voto femenino en Costa Rica. No obstante la relevancia del acontecimiento, la cobertura de la prensa escrita estuvo (posiblemente de una manera irónica) más enfocada en el vacío del control del Instituto Nacional de la Mujer (INAMU) por la partida de su directora Maureen Clark para convertirse en candidata a diputada en las elecciones de 2014. También fue importante la ausencia en la actividad de la primera mujer presidenta de la Republica, Laura Chinchilla Miranda, y la contradicción en las razones que se expusieron para esta ausencia (Mata, 29 de agosto de 2013, s.p.).

La celebración del derecho al voto de la mujer y la importancia de este hecho en el tema de los derechos políticos de las mujeres fue un punto importante que motivó a pensar en la importancia de la incursión femenina en la política electoral del país en la propia coyuntura en que esto tomó forma: la década de 1950.

A raíz del trabajo de investigación realizado para la tesis de licenciatura en Historia (Gamboa, 2012) donde se estudió el contenido de los discursos políticos que recuperaban la memoria de la Guerra Civil de 1948 durante las campañas electorales entre 1953 y 1970, es que se propone repensar la cuestión 
de la participación o la ausencia de la figura de las mujeres en esos discursos. Lo anterior pensando en el contexto de las primeras participaciones de las mujeres como electoras y aspirantes a puestos políticos. Si bien el tema de la presencia de la mujer en estos discursos políticos no fue desarrollado en dicho trabajo de investigación, en estos momentos la presencia o ausencia de la mujer en esos discursos parece un tema oportuno. El elemento que conduce a este análisis es la posibilidad de interpretar los textos desde los intereses de quienes construían esos discursos políticos: los hombres.

Ahora bien, no es del todo cierto que la imagen de la mujer estuviera ausente del discurso de los partidos políticos en las campañas electorales de 1953 y 1958. Lo que parece extraño es que, habiéndose convertido las mujeres en un caudal de votos importante, los partidos políticos no desarrollaran un discurso estratégico para ganarse a este nuevo miembro del padrón electoral, ¿significará esto que la política en la década de 1950 seguía siendo asunto de hombres?

A nivel general, la pregunta anterior podría ser contestada no solo estudiando los contenidos de los discursos sobre o de mujeres; al mismo tiempo se hace necesario analizar la construcción y reproducción de la masculinidad durante este periodo a través de sus discursos políticos. En un inicio, es posible inferir que, quizá la construcción de la masculinidad en esta época tenga que ver con la posibilidad latente de un nuevo conflicto armado (Gamboa, 2012, 231). En ese sentido, se explicaría también la presencia en esos discursos de un temor de retorno al pasado donde sí sería más importante la imagen de la mujer. Eso sí, las mujeres entraban en este discurso solamente como agentes contenedoras de la violencia masculina. Al final de cuentas ellas corrían el peligro de perder a "sus" hombres en una guerra, y por lo tanto podían, o debían evitar que esos "sus hombres" fueran nuevamente a una lucha armada.

En la campaña electoral de 1953, seenfrentaron los candidatos JoséFigueres Ferrer y Fernando Castro Cervantes. El primero, líder del Partido Liberación Nacional, principal grupo ganador de la Guerra Civil de 1948. El segundo, por su parte, intentaba constituirse con su Partido Demócrata en el representante de los grupos opositores a Figueres Ferrer. El Partido Progresista Independiente fue declarado ilegal solo unos días antes de las elecciones supuestamente por ser un partido comunista.

En el contexto de una recomposición de las relaciones sociopolíticas después de la lucha armada, precisamente la recuperación de la memoria de la Guerra Civil de 1948 será constante en los discursos políticos. Este uso estratégico del pasado sobre el conflicto tendrá como objetivo la justificación del acceso al poder por parte de unos y la misma justificación para negar ese poder a los rivales de turno, sin importar el papel jugado por unos y otros durante la guerra (Gamboa, 2012, 43). La utilización constante del discurso sobre el 48 hizo que el hecho histórico tuviera una importante variedad de interpretaciones dependiendo de los intereses con que se estuviera trayendo a colación. La 
cercanía de los hechos hicieron que en esta campaña electoral se mostrara un proceso de "acomodo" en las relaciones políticas de los principales protagonistas del conflicto, proceso en el que ya se veía la influencia del liderazgo de Figueres Ferrer, no solo como líder de los ganadores de la guerra y de la formación del Partido Liberación Nacional en 1951, sino también como el motivo por el que se comienza a generar un movimiento de oposición hacia él. Es decir, paralelamente se estaba ante el nacimiento del figuerismo y del antifiguerismo, nuevas formas del caudillismo propio de la primera mitad del siglo XX, que incluso aún en esta parte del siglo XXI encuentra algunos matices desteñidos.

En esta continuación de luchas entre vencedores y perdedores de la Guerra Civil de 1948, el único discurso político encontrado y relacionado al conflicto, en el cual que se rescató la importancia del sector femenino del electorado, lo produjo el Partido Demócrata. El 23 de junio de 1953 los demócratas titularon un espacio político pagado "Las mujeres de Costa Rica salvarán a la Patria del peligro figuerista!" (La Nación, 23 de junio de 1953, 9). El contenido del texto tiene evidentemente una clara intención de despertar ese temor al pasado muy propio de los mensajes políticos de la época. Se aseguraba que esas mujeres, que ahora iban a votar, experimentaron en su momento, durante la guerra y los dieciocho meses del gobierno posterior de Figueres Ferrer, "el dolor de las persecuciones" y "el derramamiento de la sangre entre hermanos". Por otro lado señalaba que, tuvieron que soportar "campañas de odio y rencor" en toda la década de 1940, y que por ello impedirían el "trágico regreso de Figueres", para darle un "triunfo a la causa de la tranquilidad nacional".

Esta parte del texto refiere al carácter de contenedoras de la violencia, que supuestamente tenían las mujeres, mencionado anteriormente. Este tipo de argumentos no necesariamente están ausentes del discurso dirigido a todos los sectores sociales del país. En efecto, el juego con el temor al retorno se utilizó constantemente, pero en el caso de las mujeres el estilo refuerza una imagen de tristeza y dolor más elaborada.

Sin embargo, como corolario de este papel femenino "contenedor de la violencia", aparece también un discurso sobre su papel de víctima en la guerra. En el mismo texto se les pedía a las mujeres recordar "los despojos a la propiedad privada y los asesinatos de sus esposos, sus padres y sus hijos", y "la desesperación de ver su hogar mancillado por la soldadesca en la requisa de sus esposos para ser conducidos a la cárcel, para entregarlos a tribunales inquisitoriales". En esta parte evidentemente se acentuaba la relación que las mujeres tenían con los hombres en la guerra y no como protagonistas directas de ella. Aunque sí es mencionado luego que por "primera vez en la historia de Costa Rica, damas distinguidas (fueron) encarceladas en el Buen Pastor por simple razón de odios incontrolables". Esta última referencia a la mujer en la guerra, como se verá adelante, no fue usada en otras oportunidades en la construcción de estos discursos. 
La condición de la mujer asociada a "sus hombres" no debe conducir al olvido del principal propósito de este tipo de discurso, que es poner en evidencia el carácter violento que supuestamente tenía Figueres Ferrer y su partido. Este elemento en particular hacía latente una amenaza de un retorno a la violencia del 48 en el caso de que el líder liberacionista regresara al poder político. Es decir, el discurso dirigido hacia las mujeres tenía como eje la reconstrucción de la figura de un hombre que formó parte de un pasado violento, que las mujeres debían rechazar por la relación que eso tenía con "sus hombres".

Bastante diferente es un campo pagado por una antifiguerista (La Nación, 18 de julio de 1953, 21) titulado "Están equivocados los figueristas si creen que a las mujeres nos amedrentan". La partidaria del Partido Demócrata quería señalarles a los liberacionistas que, siendo en 1948 parte de las mujeres opositoras al régimen calderonista, no se dejaron amedrentar por las amenazas de violencia. El texto es muy ilustrativo, por cuando habla de un grupo de mujeres opositoras al gobierno en 1948 y quienes se manifestaban como tales. Infiere que, a pesar de que Figueres Ferrer y su partido se adueñaron del triunfo de la Guerra Civil de 1948, no necesariamente todos esos oposicionistas a Calderón Guardia formaban parte del liberacionismo unos años después. Además, pretendía demostrar que existió en 1948 y en 1953 un grupo de mujeres con participación política, aunque no se diga directamente, ahora con más poder por su acceso al voto. Este tipo de discurso, sin embargo, lo creemos bastante excepcional cuando de recuperar la memoria de 1948 por parte de y/o para las mujeres se trata.

Ahora bien, el condicionamiento a un segundo plano (cuando no la invisibilidad) de la imagen de la mujer en los discursos políticos de la década de 1950 no calza con las luchas feministas presentes desde antes de esta década. Estamos convencidos de que la mujer no estaba (ni lo estuvo antes del voto femenino) reducida al papel de madre, esposa, hermana e hija en la política. Más bien, este tipo de discurso respondía a los intereses de los hombres, que dominaban el espacio político.

Las investigaciones realizadas sobre el tema de la adquisición del voto femenino, primero por la socióloga Macarena Barahona (1994), y luego, por la historiadora Eugenia Rodríguez (1999, 2002, 2003), demuestran que, desde antes de la reforma electoral de 1948 que incorpora el derecho al voto femenino, existieron luchas de organizaciones feministas dedicadas a alcanzar este y otros temas de igualdad de género.

Parece más creíble que los hombres, que controlaban los espacios de poder político, estaban atrapados en un juego de violencia con un punto máximo en la coyuntura de 1948. En ese caso, la relevancia de la memoria del conflicto como herramienta electoral, "los obligaba" a hacer de la política un asunto de hombres, a pesar de que las mujeres ya participaban de una manera más 
directa con su derecho al voto. Es por ello que las mujeres, en primer término, ocupaban el espacio de contenedoras de violencia en estos discursos.

Otro tipo de discurso en esta campaña electoral, si bien resalta la participación política de las mujeres, premia características de estas que no se consideran cuando se está hablando de la participación de los hombres o del "pueblo" en general. Por ejemplo, el 24 de julio de 1953 (Diario de Costa Rica, 16), con el título "Mujeres de Costa Rica, se acuerdan", los liberacionistas rescataron lo que consideraban el accionar de las mujeres antes y durante la Huelga de los Brazos Caídos en agosto de 1947. Se afirmó ahí que ese día cerca de ocho mil mujeres salieron a la calles de San José a pedirle al presidente Teodoro Picado y a los comunistas que dejaran el poder.

Lo más importante de esa manifestación, según quienes construyeron el discurso, sería su carácter no violento y la religiosidad que manifestaron las mujeres cuando participaron en dicha actividad de protesta. Se señala que le pedían al Presidente Picado "la devolución de las libertades que han sido cercenadas" por las fuerzas gubernamentales. También se mencionaba que las mujeres, antes de partir a la protesta, "se recogerán en los templos para preparar el espíritu para la gran jornada cívica”. Más adelante se indicó que en aquella ocasión las mujeres rechazaron la protección ofrecida por caballeros para evitar cualquier interpretación política de la actividad. A pesar de todo este carácter pacífico de las mujeres en huelga, al final se desató "toda la villanía, toda la bajeza y la suciedad existentes en el oscuro fondo de los hombres del Gobierno", cuando el Presidente Picado desiste de atenderlas, les dice que, "pidan un milagro a la Virgen de los Ángeles", además debieron soportar "la burla y el insulto de los militares y comunistas", y la policía les lanzaba una "afrentosa furia de sus cintarazos y sus disparos, obligándolas a buscar refugio en las casas vecinas".

En todo el discurso anteriormente señalado, es evidente que la participación política de las mujeres en el contexto del 48, que rescataba los políticos, no era para nada similar al de los hombres, como se verá más adelante. Incluso, la diferencia entre los hombres está en la manera en que se comportan ante las mujeres. El espacio físico, cuando no es el religioso, sería el mismo donde los hombres se enfrentan violentamente: las calles; empero la presencia de las mujeres le daba otro carácter a ese espacio. El irrespeto de los representantes del gobierno a ese espacio ocupado, al final de cuentas, es lo que importaba resaltar en el discurso. No es la acción de las mujeres la que interesa sino la del "villano" que formaba parte del gobierno en ese momento. La intención, más allá de querer resaltar el papel de la mujer, es el papel de los dos tipos de hombres que se involucraron en el episodio, los caballeros que se ofrecieron a proteger a las damas frente a los que representaban todo lo más negativo de la masculinidad al enfrentarse violentamente a las mujeres. No está demás señalar que, detrás de este discurso, Liberación Nacional pretendía ubicar a 
sus líderes dentro de los primeros e identificar a los miembros del Partido Demócrata con el grupo de los villanos.

En la campaña electoral de 1958, en la que se enfrentaron por la presidencia Francisco Orlich de Liberación Nacional, Mario Echandi por el Partido Unión Nacional y Jorge Rossi por el Partido Independiente, la idea de la participación política de las mujeres no perderá ese carácter pasivo y relacionado al de "sus" hombres del 48. Incluso la invisibilización de la mujer sería mayor porque se tuvo acceso a menos textos relacionados a las mujeres, por la menor cantidad de ellos en los periódicos, respecto a cuatro años atrás. Eso a pesar que la cantidad de discursos analizados para esta campaña electoral fue mayor que para el caso de 1953 (Gamboa, 2012, 46 y 85). El estudio de estos discursos refuerza la idea de que las mujeres, aunque tenían sus propias experiencias de la Guerra Civil de 1948, no eran parte de un espacio muy bien trabajado por los candidatos a los puestos políticos. Lo anterior, a pesar de que diez años después del conflicto, como parte del electorado y del peso político que con ello tenían, las mujeres debían tener un criterio propio para decidir cómo canalizar electoralmente su experiencia del conflicto. El tema de la mujer en la política parecía ser un asunto que aún pretendían decidir los hombres.

En algún caso de estos discursos, se encontró el de una mujer liberacionista. Esta mujer se vio tentada en algún momento de separarse del Partido Liberación Nacional, pero afirma que "como madre de un soldado de Liberación, caído en la Batalla de El Tejar en 1948, yo no puedo alejarme de sus filas" (La Republica, 4 de junio de 1957, 20).

Otro caso es el de una madre que protesta por el uso que los echandistas hicieron de la imagen fotográfica de su hijo, muerto durante un enfrentamiento armado, en un espacio político pagado. El carácter de víctima y de héroe manipulado por los opositores, fue usado por los liberacionistas para asegurar que Echandi y su partido no sabían respetar el dolor de una madre por el hijo muerto (La República, 12 de setiembre de 1957, 23) y (La República, 14 de setiembre de 1957, 13).

A pesar que estos son los únicos casos sobre mujeres con alguna memoria de la guerra de 1948 encontrados en los textos estudiados para la campaña electoral de 1958, no debería significar que necesariamente la mujer como sujeto político con derecho al voto no fuera importante en estas campañas políticas. En el proceso de identificación de las fuentes utilizadas se reconoció una importante cantidad de espacios políticos pagados dirigidos a las mujeres de unos u otros partidos, pero que no tenían ninguna alusión directa al conflicto de 1948. Como este trabajo se enfoca en el análisis de la construcción de identidades de género en los discursos relacionados directamente a la Guerra Civil de 1948, se decidió no hacer uso de estos textos. Eso sí, cabe señalar que, de todas formas, en su mayoría estos textos estaban dirigidos a promover la organización de los sectores femeninos en cada partido. La presencia 
de este tipo de espacio político pagado tuvo mayor relevancia en la campaña electoral de 1953 por el Partido Demócrata, que sin mucho recurso discursivo invitaba al "ala femenina" de ese partido a organizarse para las elecciones (La Nación, 24 de junio de 1953, 6). Estos textos propagandísticos de todas formas carecían de un discurso sobre las diferentes reivindicaciones de género que las mujeres pudieran tener.

\section{Los hombres en los discursos políticos sobre el 48: una guerra de hombres}

Ya que los discursos políticos sobre las mujeres fueron construidos en su mayor parte por hombres, posiblemente sea bueno acercarse también a ellos. En ese sentido, es importante buscar las razones y las intenciones de esos discursos, pensando que pueden convertirse en un buen ejercicio para estudiar en la construcción de la masculinidad algún reflejo a su vez de la construcción de la feminidad en la política costarricense en la década en la cual la mujer tuvo acceso al voto.

Como fue señalado, la Guerra Civil de 1948 originó una serie de cambios político institucionales que determinarían a su vez la estructura socioeconómica y política en la segunda mitad del siglo XX. En cuanto a la construcción de identidades masculinas y femeninas en esta segunda parte del siglo XX, González Ortega (2005, 27-27) asegura que, después de la Guerra Civil de 1948 la clase política del país inició un proceso de "madurez" referente a la construcción de la masculinidad. La "capacidad de autogobernarse", expresada en la consolidación del dominio de la naturaleza, (en la cual González Ortega hace una analogía con el dominio sobre lo femenino), sería un reflejo de la madurez de la masculinidad contrapuesta a la inmadurez anterior a la coyuntura conflictiva de 1948.

De ahí se infiere una ruptura: un antes y un después con el conflicto armado; ya no solo en factores jurídicos y estructurales, sino en la construcción de nuevas identidades políticas. Es cierto que con la aparición de nuevos actores políticos y los cambios que ellos impulsaron, la década de 1940 puede, y debe ser vista como un punto de ruptura. No obstante, la presencia de continuidades entre las dos décadas en cuanto a la forma de hacer política, necesariamente lleva a cuestionar un cambio sustancial, por lo menos en la construcción de lo masculino y lo femenino en la década de 1950.

Lo anterior, porque los discursos políticos de las campañas electorales de la década de 1950 muestran aspectos que se parecen mucho a la forma de hacer política en la década anterior; y esa similitud es más evidente cuando de hablar de masculinidades se trata. El caudillismo, el personalismo y el patriarcalismo, muy propios del quehacer político de la primera mitad de siglo XX, son elementos que no dejan de aparecer en el vocabulario político después de 1948. De ahí que estos discursos deben dar algunas pistas sobre cómo 
se manejó la masculinidad en un contexto donde la mujer ya tenía derecho a votar, e iniciaba un proceso de inclusión política real, el mismo que aún no ha concluido del todo.

En realidad, lo que más se reproduce en estos discursos por cada partido político es la intención de mostrar al votante que el contrincante de turno hace una política violenta similar a la caracterizada en la década de 1940. Incluso, según este discurso, serían los mismos hombres los que usaban la violencia constantemente para imponer sus intereses políticos. En ese sentido, las intenciones de los partidos no se alejan del temor al retorno al pasado que se señaló antes. Existe ahí un juego discursivo donde la Guerra Civil de 1948 es vista como un punto de ruptura, pero al mismo tiempo se denuncian elementos que se dicen propios del periodo anterior al 48 y que deben ser evitados a toda costa, incluso con el discurso irónico del uso de la violencia, si fuera necesario.

Empecemos con los discursos que tienen en común elementos propios del caudillismo, personalismo y patriarcalismo. En las dos campañas electorales estudiadas, el Partido Liberación Nacional siempre intentó encontrar en sus partidos contrincantes elementos que pudieran ser relacionados con el periodo anterior al 48. Esta época, que los liberacionistas consideraban superada, o necesaria de superar, era llamada en sus discursos el "periodo de los ocho años caldero-comunistas", y se asociaba con una serie de aspectos de violencia no propios de la cultura costarricense (Gamboa, 2012, 62). En ese sentido, se debe entender que el triunfo de Figueres Ferrer y su gente en la Guerra Civil de 1948 le daba algún derecho moral para justificar su ascenso al poder y la negación del mismo a los llamados "vencidos".

De este modo, para Liberación Nacional, hacer un juego político electoral construido con base en una imagen idealizada y contrapuesta entre las dos épocas antes y después del 48 se convirtió en estrategia continua. Esta herramienta electoral en el discurso se completaba con la intención de identificar personajes de la época superada en los partidos rivales. Así, en algunos de los discursos liberacionistas, el proceso político en las décadas de 1940 y 1950 fue visto por etapas. Primero hay una anterior a la Guerra Civil de 1948, esta se encontaría caracterizada por un régimen opresivo y de una lucha opositora. Luego estaría "la guerra de liberación" llevada a cabo por Figueres Ferrer y su gente, para acabar con el periodo de los calderocomunistas en el poder, y después la etapa de construcción del nuevo país, y de la necesidad de evitar que Calderón Guardia y sus partidarios regresaran al poder político. En ese discurso Figueres Ferrer sería visto como el "artífice del gesto de rebeldía que condujo a la Costa Rica de hoy", a la Costa Rica posterior a la guerra (La República, 17 de julio de 1953, 9).

La doble intención de Liberación Nacional se hace latente también en la campaña electoral de 1953, cuando se aseguraba que el candidato opositor, 90 Castro Cervantes "en pacto secreto con Calderón Guardia ha ofrecido dejar en 
absoluta libertad, a los delincuentes que cometieron los más graves crímenes contra la comunidad costarricense" (Diario de Costa Rica, 12 de julio de 1953, 9). El discurso liberacionista intentaba con ello crear un temor de un retorno a esos hechos, a la vez que aseguraba que detrás de sus contrincantes se escondían las intenciones de los calderonistas.

En la campaña electoral de 1958, el estilo de Liberación Nacional de hacer política no varió mucho. La comparación entre épocas y la participación de los líderes de unos y otros sería la misma. En algunos de estos discursos, Liberación Nacional creó un ambiente de inseguridad por la posible influencia de calderonistas y comunistas en el Partido Unión Nacional, mientras resaltaba el mismo carácter de ruptura con la Guerra Civil de 1948 (La República, 10 de diciembre de 1957, 17).

Por su parte, los partidos opositores a Liberación Nacional tendieron a utilizar un discurso igualmente de ruptura en el 48, con la salvedad de considerarla un acto violento inventado por Figueres Ferrer solamente para conseguir sus intereses personales. Hay en esos discursos una alusión a un pasado mejor, antes de la llegada de Figueres Ferrer y su guerra. La ideología de esta oposición al figuerismo estaría idealmente relacionada a los valores de los grandes gobiernos de la primera parte del siglo XX (La Nación, 12 de diciembre de 1956, 34).

El reacomodo de las relaciones políticas antes mencionada y la consolidación de la figura política de Figueres Ferrer convirtió a este en el primer objetivo de los discursos del Partido Demócrata en 1953 y del Unión Nacional en 1958. El accionar de Figueres Ferrer se tornó en esos discursos en la "representación de la violencia, la revolución, la lucha armada entre hermanos" (Diario de Costa Rica, 11 de julio de 1953, 10). También el mismo juego del temor al retorno de un pasado violento estaba presente en este discurso antifiguerista cuando, por ejemplo, el Partido Demócrata intentó hacer una relación entre su partido y la garantía de un gobierno de paz, mientras que aseguraba que Figueres Ferrer representaba la posibilidad de una continuación de la violencia política (Diario de Costa Rica, 24 de julio de 1953, 11).

Del mismo modo, en la campaña de 1958 el tono no variaría mucho, y la llamada "lucha de la oposición" fue vista como una lucha contra el figuerismo, mientras que se denominaba al candidato del Partido Unión Nacional, Mario Echandi como un caudillo a la misma altura que los anteriores a la Guerra Civil de 1948 (La Nación, 1956, 4 de noviembre de 1956, 58), y también el mejor oposicionista a Figueres Ferrer (La Nación, 17 de enero de 1957, 16).

En esta campaña electoral, aunque el Partido Unión Nacional le apostaba a un discurso de "reconciliación nacional" (Gamboa, 2012, 103) buscando agrupar a todos los sectores perdedores de la Guerra Civil de 1948, también usó otro discurso sobre la persecución política y la violencia figuerista para intentar restar el valor moral del mismo triunfo de Figueres Ferrer en el conflicto armado. 
Uno de los aspectos más interesantes de esta construcción de la masculinidad política presente en los discursos políticos con alguna relación a la Guerra Civil de 1948, es la incorporación en estos del expresidente fallecido, León Cortés Castro. Esta reconstrucción de la figura de León Cortésy su vínculo con la política de la década de 1950 sería más utilizada por Liberación Nacional. Las principales intenciones del liberacionismo con este recurso fue, en primer lugar, hacer de León Cortés una víctima más del régimen de los "ocho años"2, y segundo, crear un paralelismo y un continuismo entre el caudillo anterior a la Guerra Civil de 1948 y el caudillo de la guerra y líder liberacionista, Figueres Ferrer.

En sus discursos, Liberación Nacional ubicaba a León Cortés entre las víctimas de la Guerra Civil de 1948, aunque la muerte del caudillo hubiera ocurrido dos años antes del conflicto armado (Diario de Costa Rica, 1953, 25 de julio de 1953, 6,7). El ejercicio liberacionista tenía algún sentido, si entendemos que en estas campañas electorales para Figueres Ferrer y su gente fue muy importante construir una imagen ambivalente entre víctimas y héroes. En efecto, Liberación Nacional hizo de sus campañas electorales un espacio de idealización de sus caídos durante la Guerra Civil, haciendo un doble discurso sobre el carácter heroico de su muerte y la condición de víctimas que les daba el régimen opresor de los "ocho años", causa principal de la lucha armada. En ese sentido, la figura de León Cortés, aunque en principio calzaba solamente con el carácter de víctima, tendía luego a confundirse entre los héroes liberacionistas.

Para los liberacionistas el vínculo Cortés-Figueres estaría en el origen del movimiento de oposición al calderonismo, que inició Cortés en 1943 y finalizó Figueres en 1948. Según este discurso de Liberación Nacional, en 1943, el pueblo de Costa Rica reunido en la ciudad de San José, mucho más pequeña en ese entonces, tenía un entusiasmo político general por León Cortés, en un contexto donde no existía libertad individual. Este último aspecto era resultado del gobierno de Calderón Guardia. El apoyo popular hacia León Cortés, no tendría el resultado esperado, porque "aquellos eran otros tiempos. Y los hombres del gobierno habían dicho que León Cortés no sería presidente" (La República, 15 de mayo de 1957, 2). Lo que fue considerado en ese momento y resaltado por Liberación Nacional en su campaña de 1958 como un claro fraude electoral, convertía a León Cortés en una víctima y en el primer opositor al calderonismo. Aparte le servía para ubicar a Figueres Ferrer como el continuador de ese caudillismo de oposición. De igual forma, estaba haciendo el mismo ejercicio de ruptura temporal entre lo que era Costa Rica antes de la coyuntura de 1948 y lo que era después.

Para Manuel Solís Avendaño (2006, 385-392) sí hubo cambios en la forma de hacer política después de la Guerra Civil de 1948, los cuales se expresaron de 1946, Liberación Nacional trató de culpar de su muerte a los calderonistas y comunistas. 
en el tipo de relaciones entre los caudillos. Sin embargo, Solís también considera que estas modificaciones se hicieron dentro de un mismo estilo político, propio de la primera parte del siglo XX en el país. En ese sentido, los cambios en las relaciones antes de la Guerra Civil de 1948, entre León Cortés y Calderón Guardia, entre este último y los comunistas, y a su vez entre estos y la Iglesia Católica, no tendrían mucha diferencia con respecto al tipo de relaciones cambiantes que se dan después de 1948 entre Figueres Ferrer y Ulate Blanco, entre este último y Calderón Guardia, entre este y los comunistas, por mencionar los ejemplos más significativos.

A raíz de lo expuesto en los párrafos anteriores, se puede asegurar que durante las campañas electorales de 1953 y 1958 hubo luchas personalistas con un carácter caudillista muy fuerte. Al igual que antes de la Guerra Civil de 1948, el espacio político estaba siendo ocupado por hombres que debían resolver sus problemas, no solo políticos, sino también de reconstrucción de la identidad masculina. Los candidatos estaban llevando a sus discursos algunos de los mismos enfrentamientos anteriores a la coyuntura de 1948, para mezclarlos con sus propias luchas originadas en el contexto de la Guerra Civil de 1948. Evidentemente, ese ejercicio se convertía en la necesidad de legitimar el enfrentamiento armado en 1948 y el posterior derecho al poder político que supuestamente el resultado del conflicto les daba, según la lectura que se hiciera desde una u otra agrupación política en disputa. Esto porque los ganadores del conflicto se sentían con ese derecho asegurado, y prácticamente lo consideraban automático, mientras que los perdedores en 1948 aseguraban haber sido víctimas de las ambiciones de Figueres Ferrer y su gente, y por ello el carácter de reivindicación política que exponían en sus discursos (Gamboa, 2012, 185).

Lo cierto es que, en los discursos políticos, la madurez de los protagonistas en la década de 1950 no parece estar muy presente en esta guerra verbal. Durante la campaña electoral de 1958, Liberación Nacional al mismo tiempo que hacía una idealización de la Guerra Civil, y sobre todo de la participación en ella de Figueres Ferrer y el candidato presidencial en ese momento, Francisco Orlich, también se ocupaba de hacer una comparación de las actuaciones de estos con las del candidato opositor, Mario Echandi. Es este juego de discursos un espacio donde se reproducen las masculinidades ideales del político vinculado a los "grandes hombres" del periodo anterior a 1948, y merecedores de esa categoría por su papel en el conflicto armado.

Por ejemplo, en un espacio político pagado titulado "Cantando sin guitarra" (La República, 4 de junio de 1957, 6), Liberación Nacional descalificaba el discurso de sus oponentes sobre la necesidad de defender la limpieza de las elecciones que se avecinaban. Los liberacionistas consideraban que tanto Ulate Blanco como el candidato Mario Echandi "ya aburren con ese cuentito y nadie les hace caso [...] por qué no lo hicieron en el 48?". También en varios de los textos para referirse a la actitud propiamente de Echandi realizaron comparaciones entre la participación de este y la de Figueres y Orlich (La República, 
27 de marzo de 1957, 27) y (La República, 12 de enero de 1958, 19). En estos discursos la imagen de unos y otros estaría determinada directamente por el uso de las armas en la guerra. Incluso, en estos textos los liberacionistas aseguran que mientras Figueres y Orlich estaban en la guerra, Echandi se ocultaba en el techo de una casa.

Las características idealizadas de los líderes políticos según este tipo de discursos estarían determinadas por componentes considerados muy masculinos en la época, como el enfrentamiento armado, la valentía y la osadía de los caudillos, así como de los elementos opuestos: la supuesta cobardía de Echandi. Pero además está presente este componente de mofa y humillación que según Alfonso González Ortega $(2005,18)$ tenía que ver con el honor de un caballero y la posibilidad de ser ofendido. González considera que algunos hombres después de la Guerra Civil de 1948 sufrieron la humillación por no haber estado al frente de batalla empuñando un arma. Un ejemplo de ello al parecer le sucedió a un Presidente Municipal en el sur del país durante el gobierno de Ulate Blanco (1949-1953), quien se queja ante el Presidente de la República por los constantes atropellos recibidos de parte del agente policial, al parecer por no haber participado directamente en la guerra. González asegura que el ofendido se siente en la obligación de defender su honor. Podemos inferir que al burlarse de Echandi, los liberacionistas perseguían también ofender el honor del ahora candidato presidencial.

Por su parte, los opositores a Figueres Ferrer también utilizaron discursos tendientes a ridiculizar a sus adversarios, al mismo tiempo que se trataba de señalar los aspectos más negativos de los liberacionistas, como se muestra en el ejemplo de la Imagen 1.

En este caso en particular, los demócratas consideraban posible hacer una relación entre Figueres Ferrer y su gente con el personaje de la película "El Siete Machos", protagonizada en 1950 por el actor comediante mexicano Mario Moreno "Cantinflas". De esta forma, de una manera también burlesca, los demócratas trataban de demostrar que Figueres era el líder de una banda de malhechores, que haciendo uso de la violencia, crearon las condiciones institucionales para poder beneficiarse personalmente.

A nivel general, el estudio de los discursos políticos desde una perspectiva de los intereses electorales de quienes los estaban construyendo, permite observar un espacio político ocupado por hombres. Estos personajes estaban usando los mismos recursos anteriores a 1948 para resolver también los mismos problemas de identidad política y masculina anteriores a la Guerra Civil de 1948 y la incorporación del derecho al voto femenino. Como bien lo señala González Ortega, a pesar de que tradicionalmente se ve un cambio en cultura político-electoral del país, con las reformas electorales y la abolición del ejército, también es cierto que elementos como enfrentamientos armados, traiciones 94 políticas, golpes de Estado, no "desaparecieron del imaginario nacional, [ni] del 


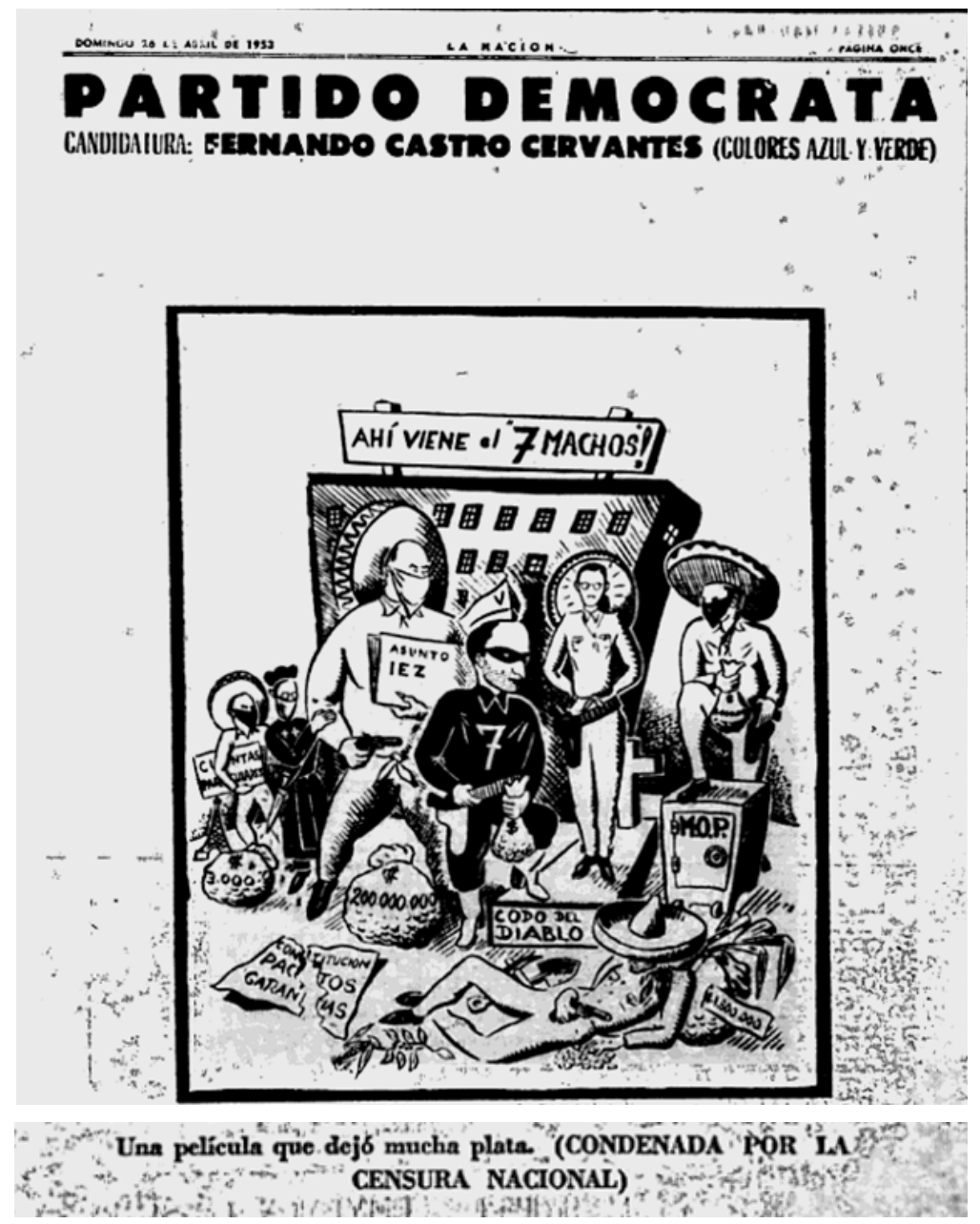

Imagen 1. Espacio político pagado por el Partido Demócrata en campaña de 1953. Fuente: La Nación, 26 de mayo de 1953. p. 11.

vocabulario de la prensa y de los discursos políticos" $(2005,13)$. Todos estos elementos formaban parte del espacio político manejado por hombres y para hombres. Aunque parecen claros los esfuerzos de los hombres de la política por mantenerse al margen de la violencia, también es cierto que en muchas ocasiones sacaban a relucir sus intenciones de responder con violencia los ataques de sus enemigos de turno.

\section{Conclusiones}

El papel de las mujeres rescatado en los discursos políticos durante las campañas electorales de 1953 y 1958 si bien no las invisibiliza del todo, no llega a ocupar un espacio determinante, si se compara con el protagonismo de los hombres que es recuperado en estos discursos políticos. Lo anterior a pesar de que este tipo de campañas electorales se dan en un contexto donde 
se supone las mujeres han adquirido un peso político relevante a raíz de su derecho al voto recién alcanzado. En primera instancia, uno de los motivos para que sucediera lo anterior debió de ser la construcción cultural sobre los roles de género que imperaban en la época (Rodríguez, 2003b, 9-13). La mujer estaba circunscrita a los espacios del hogar y de lo privado. En ese sentido, la adquisición del derecho del voto era un paso para que la mujer se incorporara a los espacios de acción pública, pero ese ha sido un proceso lento que aún no termina de consolidarse, aunque las luchas de y con las feministas hayan obtenido muy importantes éxitos en ese proceso.

Volviendo a la década de 1950, a pesar de que las mujeres tenían su propia experiencia relacionada a la Guerra Civil de 1948, el carácter masculino de los discursos políticos rescatan solo las condiciones de esa experiencia que estuvieran ligadas a la experiencia de los hombres cercanos a ellas. Es por eso que es válido asegurar que en estos discursos no se hace referencia directa a las mujeres, sino a las madres, esposas, hermanas e hijas de los hombres de la guerra.

Toda esta construcción de género de los discursos sobre las mujeres y los hombres, tendrían su origen en algunas de las particularidades de hacer política en el periodo posterior a la Guerra Civil de 1948. En primer lugar, las principales intensiones de los candidatos eran, por un lado, hacer del temor del retorno a un pasado de violencia una herramienta psicológica útil y efectiva para legitimar el acceso al poder, mientras se justificaba también la negación de ese mismo poder a los contrincantes. En ese sentido, era más sencillo dirigir un discurso hacia las mujeres, donde se planteaba la amenaza latente de ese retorno violento en caso de que el adversario lograra ganar las elecciones.

Por otro lado, hacer del juego político un asunto de hombres debió responder también a las necesidades de identidad política que los protagonistas debían llenar. Este aspecto en particular se evidencia cuando se identifican discursos que pretenden hacer un vínculo entre caudillos anteriores a la coyuntura de 1948 y los caudillos surgidos de la Guerra Civil de 1948. La intención es legitimar su liderazgo y de nuevo, su ascenso al poder, construyendo una línea imaginaria entre la experiencia y la lucha del líder ya legitimado en el pasado. También se pudo observar que la experiencia particular por cada sujeto durante la Guerra Civil, junto con la forma en que se construyen las identidades de masculinidad en la época, permitían hacer comparaciones de tipo burlesco entre las aptitudes asumidas por unos y otros durante el conflicto armado. Este último aspecto llama la atención porque a nivel general los discursos tratan de mostrar los aspectos más negativos de la experiencia vivida durante la Guerra Civil de 1948; no obstante esta posición, muestra que algunos hombres consideraban que se habían hecho más hombres empuñando un arma contra otro costarricense. 
Sirva la anterior afirmación en primera lugar, para considerar que los discursos políticos en la década de 1948 evidencian el carácter violento de la política costarricense en la década de 1950. Es claro, eso sí, que la amenaza de un nuevo conflicto armado se quedó, al final de cuentas, la mayor de las veces en este tipo de discurso, pero sus protagonistas también dieron muchas razones y evidencias para hacer creer que en cualquier momento resolvían sus problemas nuevamente por medio de las armas.

A partir de esta imagen que queda de las luchas entre hombres de la política en la década de 1950, quizá sea posible asegurar que la incorporación de las mujeres a la política por medio de su derecho al voto (sin desmeritar el valor real que ello significara) no dejaba de ser una herramienta más con la que unos hombres pretendían vencer a otros hombres. El verdadero significado de esta reforma electoral se lo han dado las mismas mujeres, quienes a través de los años (antes y después del voto femenino) se han ganado el derecho a la participación política por cuenta propia y no como un derecho otorgado.

\section{Referencias}

A las mujeres castristas. (1953, 24 de junio). La Nación, p. 6.

Así trata el Echandismo a los héroes! (1957, 14 de setiembre). La República, p. 13.

Barahona, Macarena. (1994). Las sufragistas en Costa Rica. San José: Editorial de la Universidad de Costa Rica.

Calle Blancos de Goicoechea con el verde blanco y verde. (1953, 17 de julio). La República, p. 9.

Cantando sin guitarra. (1957, 4 de junio). La República, p. 6.

Crímenes, robos y sacrilegios calderocomunistas. (1953, 12 de julio). Diario de Costa Rica, p. 9.

Destruya al figuerismo con Mario Echandi. (1957, 17 de enero). La Nación, p. 16.

El pueblo no quiere ser redentor de los ajenos, sino de sí mismo. (1956, 12 de diciembre). La Nación, p. 34.

En Costa Rica ha sucedido algo más que un cambio de gobierno. (1957, 10 de diciembre). La República, p. 17.

Están equivocados los figueristas si creen que a las mujeres nos amedrentan. (1953, 18 de julio). La Nación, p. 21.

Gamboa, Manuel. (2012). Los discursos políticos durante las campañas electorales entre 1953 y 1970 en Costa Rica: la memoria de la Guerra Civil de 1948. Tesis de Licenciatura en Historia, Universidad de Costa Rica, San José, Costa Rica. 
González, Alfonso. (2005). Mujeres y hombres de la posguerra costarricense (1950-1960). San José: Editorial de la Universidad de Costa Rica.

Las garantías presidenciales de paz, orden y libertad electoral. (1953, 24 de julio). Diario de Costa Rica, p. 11.

¡Las mujeres de Costa Rica salvarán a la Patria del peligro figuerista! (1953, 23 de junio). La Nación, p. 9.

Mata, Esteban. (2013, 29 de agosto). Chinchilla ausente en celebración del 60 aniversario de participación de la mujer en la política. La Nación. Recuperado el 28 de octubre del 2013 de http://www.nacion.com/nacional/politica/ Chinchilla-ausente-aniversario-participacion-politica_0_1362863772.html

Mujeres de Costa Rica, se acuerdan. (1953, 24 de julio). Diario de Costa Rica, p. 16.

Mujeres de Costa Rica. (1953, 11 de julio). Diario de Costa Rica, p. 10.

Nunca jamás. (1958,12 de enero). La República, p. 19.

Partido Liberación Nacional. (1957, 4 de junio). La República, p. 20.

Partido Unión Nacional inicia su campaña regular de prensa y radio. (1956, 4 de noviembre). La Nación, p. 58.

Por la memoria de estos mártires. (1953, 25 de julio). Diario de Costa Rica, pp. 6-7.

Protesta una madre por una publicación echandista. (1957, 12 de setiembre). La República, p. 23.

Recado para el pueblo. (1957, 27 de marzo). La República, p. 27.

Rodríguez, Eugenia. (1999). Los discursos sobre la participación de las mujeres en la política en Costa Rica (1910-1949). Revista Parlamentaria, 7 (1), pp. 85-122.

Rodríguez, Eugenia. (2002). La lucha por el sufragio femenino en Costa Rica (1890-1949). En Rodríguez, Eugenia. (Ed.). Un siglo de luchas femeninas en América Latina. San José: Editorial de la Universidad de Costa Rica.

Rodríguez, Eugenia. (2003a). Dotar del voto a la mujer ¿Por qué no se aprobó el voto femenino en Costa Rica hasta 1949? Serie Cuadernos de Historia de las Instituciones en Costa Rica, Escuela de Historia, No. 3. San José: Editorial Universidad de Costa Rica.

Rodríguez, Eugenia. (2003b). Los discursos sobre la familia y las relaciones de género en Costa Rica (1890-1930). Serie Cuadernos de Historia de las 
Instituciones en Costa Rica, Escuela de Historia, No. 2. San José: Editorial Universidad de Costa Rica.

Rovira, Jorge. (1983). Estado y Política económica en Costa Rica. 1948-1970. Segunda Edición. San José: Editorial Porvenir.

Solís, Manuel. (2006). La institucionalidad ajena: Ios años cuarenta y el fin de siglo. San José: Editorial de la Universidad de Costa Rica.

Una fecha gloriosa. (1957, 15 de mayo). La República, p. 2. 